\title{
Detection of an Unidentified Extended Gamma-ray Source Close to the Galactic Supernova Remnant 3C 400.2
}

Tülün Ergin* TUBITAK Space Technologies Research Institute, Ankara, Turkey

E-mail: tulun.ergin@tubitak.gov.tr

Aytap Sezer, Avrasya University, Trabzon, Turkey

Ryo Yamazaki, Aoyama Gakuin University, Fuchinobe, Japan

Hidetoshi Sano, Nagoya University, Nagoya, Japan

Yasuo Fukui, Nagoya University, Nagoya, Japan

Shuta Tanaka, Konan University, Kobe, Japan

\begin{abstract}
A new extended gamma-ray source (PS J1934.5+1845) was detected with a significance of $\sim 13 \sigma$ at a location of $1^{\circ} .83$ away from the radio location of the Galactic supernova remnant $3 \mathrm{C} 400.2$ using about 9 years of Fermi-LAT data. The $68 \%$ containment radius of PS J1934.5+1845's extension was found to be $0^{\circ} .61$ and PS J1934.5+1845 is showing a power-law type spectrum with a spectral index of $\sim 2.38$. In this presentation we will summarize the gamma-ray analysis methods and report on the analysis results related to the extension and spectrum of PS J1934.5+1845.
\end{abstract}

7th Fermi Symposium 2017

15-20 October 2017

Garmisch-Partenkirchen, Germany

\footnotetext{
*Speaker.
} 


\section{Introduction}

While searching for gamma-ray emission from the mixed-morphology (MM; [5]) supernova remnant (SNR) 3C 400.2 (G53.6-2.2) [3], we detected a new extended unidentified gamma-ray source which we named as PS J1934.5+1845 after its best-fitted location. We start this paper by shortly explaining the analysis of Fermi-LAT data in Section 2. In Section 3, we summarize the analysis results for PS J1934.5+1845 and present our conclusions in Section 4.

\section{Data Reduction \& Analysis}

Using the analysis toolkit fermipy ${ }^{1}$ we analyzed Fermi-LAT gamma-ray data from the time period of 2008-08-04 to 2017-02-06 for events within the energy range of $200 \mathrm{MeV}-300 \mathrm{GeV}$. Using gt select of Fermi Science Tools (FST) ${ }^{2}$, we selected Fermi-LAT Pass 8 'Source' class and front+back type events, which come from zenith angles smaller than $90^{\circ}$ and from within a circular region of interest (ROI) with a radius of $30^{\circ}$ centered at the radio position of $3 \mathrm{C} 400.2$. The maximum likelihood fitting method [4] was employed on the spatially and spectrally binned data and used the instrument function P8R2_SOURCE_V6. The gamma-ray background model contains Galactic diffuse sources ( $g l l_{-} i e m_{-} v 6$. fits) and isotropic sources (iso_P8R2_SOURCE_V6_v06.txt). It also includes all point-like and extended sources from the 3rd Fermi-LAT source catalog (3FGL) [1] located within a $15^{\circ} \times 15^{\circ}$ region centered at the ROI center. Freed normalization parameters of sources that are within $3^{\circ}$ of ROI center. Freed all parameters of the diffuse Galactic emission and the isotropic component. All sources with TS $>10$ are set free and all sources with TS $<10$ are fixed.

The initial $\mathrm{TS}^{3}$ map was produced for a $10^{\circ} \times 10^{\circ}$ analysis region using this model showing gamma-ray excess close to the location of $3 \mathrm{C} 400.2$ and at other locations within the analysis region. In order to obtain the exact TS value and location of the excess regions, we used an iterative source-finding algorithm in fermipy, called find_sources:

- Takes peak detection on a TS map to find new source candidates. The algorithm identified peaks with a significance threshold value higher than $3 \sigma$ and taking an angular distance of at least 1.5 from a higher amplitude peak in the map.

- It orders the peaks by their TS values and adds a source at each peak starting from the highest TS peak.

- Then it sets the source position by fitting a 2D parabola to the log-likelihood surface around the peak maximum.

- After adding each source, having a significance value above $5 \sigma$, it re-fits the spectral parameters of that source.

- With this algorithm, we identified nine new sources within the analysis region.

- The algorithm also listed the sources with a significance between $3 \sigma$ and $5 \sigma$. One of these sources, PS J1938.6+1722, was within the $95 \%$ confidence radius of the $3 \mathrm{C} 400.2$ position. So, the name of PS J1938.6+1722 is used for 3C 400.2.

\footnotetext{
${ }^{1} \mathrm{http}: / /$ fermipy.readthedocs.io/en/latest/index.html

${ }^{2}$ https://fermi.gsfc.nasa.gov/ssc/data/analysis/software/

${ }^{3}$ Test statistics (TS) indicate that the null hypothesis (maximum likelihood value for a model without an additional source) is incorrect.
} 
As a next step we analyzed the closer vicinity of $3 \mathrm{C} 400.2$ by taking an analysis region of $5^{\circ} \times 5^{\circ}$. We followed the same analysis procedure as described for the $10^{\circ} \times 10^{\circ}$ analysis region, except that we added two sources into the background model: PS J1934.5+1845, being the only bright gamma-ray source having a significance above $5 \sigma$ within the analysis region, and PS J1938.6+1722, representing 3C 400.2.
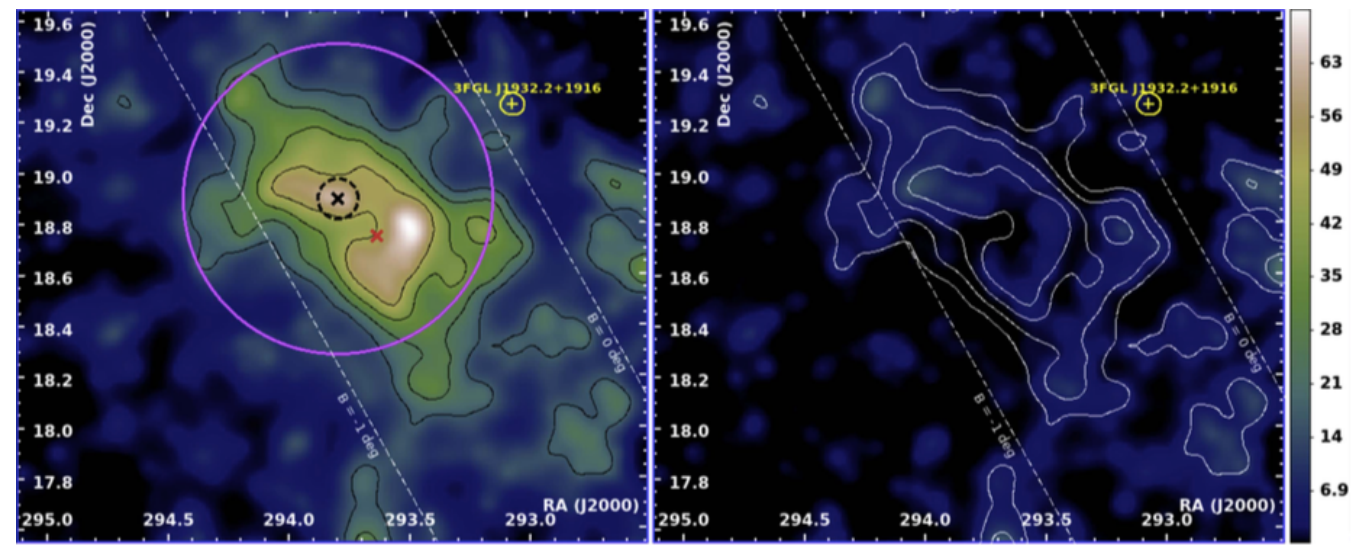

Figure 1: TS map of the gamma-ray emission, where PS J1934.5+1845 was not included in the background model (left panel). The initial spatial model of the source was taken as point-like, where its location was chosen as the red cross shown in the left panel. The best-fit position of the point-like source is shown with a black cross, and its positional error at a 95\% confidence level is shown as a black dashed circle. The magenta solid circle represents the best-fit extension found using a radial Gaussian model centered around the best-fit position of PS J1934.5+1845. The right panel shows the gamma-ray TS map, where PS J1934.5+1845 was included as a point-like gamma-ray source in the background model. The black and white contours on both panels are the levels of the TS values, which are 16, 25, 36, 49, 69, and a gamma-ray source from 3FGL [1] is shown in yellow, together with its positional error circle. The white dashed lines correspond to the Galactic latitudes of $-1^{\circ}$ and $0^{\circ}$.

\section{Analysis Results}

\subsection{Detection \& Localization}

PS J1934.5+1845, was detected at a location of 1.83 away from the radio location of $3 \mathrm{C} 400.2$. The detection significance was found to be $\sim 13 \sigma$ (i.e., $\mathrm{TS}=161$ ), assuming PS J1934.5+1845 as a point-like source during the source search procedure. Using the localize method of fermipy, the best-fitted position for PS J1934.5+1845 was found to be R.A. $(\mathrm{J} 2000)=293^{\circ} .79 \pm 0^{\circ} .08$ and decl. $(\mathrm{J} 2000)=18^{\circ} .90 \pm 0^{\circ} .08$ (R.A.

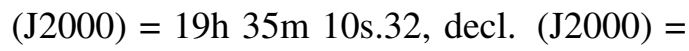
$\left.18^{\circ} 54^{\prime} 07^{\prime \prime} .20\right)$.

We checked 3FGL [1] and the 3rd catalog of hard Fermi-LAT sources (3FHL) [2] to find possible counterparts for PS J1934.5+1845. Figure 1 shows the TS map

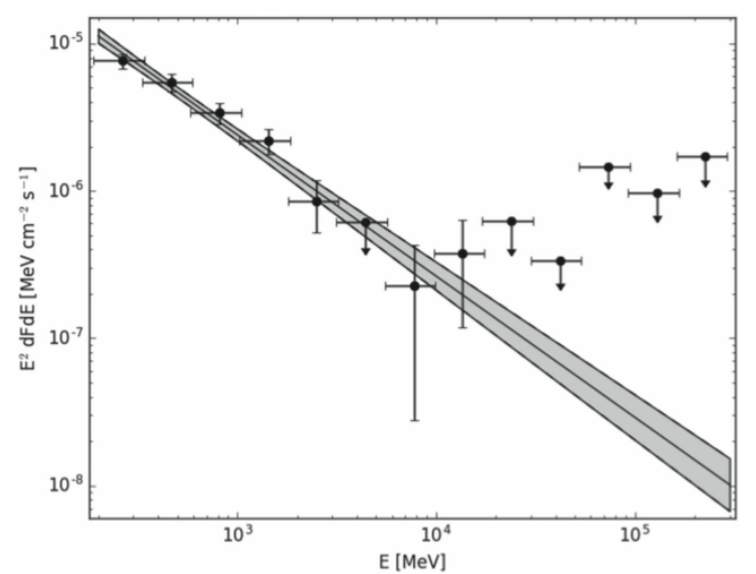

Figure 2: Gamma-ray spectral energy distribution of PS J1934.5+1845 assuming it to be an extended source. The shaded region represents the model flux and its statistical errors obtained from fitting a PL-type spectrum to the given spectral data. 
of PS J1934.5+1845. Fermi-LAT sources from 3FGL [1] as yellow markers. We could not find any counterparts for SourceA in 3FGL catalog.

\subsection{Extension Measurements}

We used two models to parameterize the extended gamma- ray emission morphology of PS J1934.5+1845, disk and radial Gaussian models, where the width and location of the centers of the models were calculated by the extension method of fermipy. To detect the extension of a source, we used the TS of the extension $\left(\mathrm{TS}_{\text {ext }}\right.$ ) parameter, which is the likelihood ratio comparing the likelihood for being a point-like source $\left(\mathrm{L}_{p t}\right)$ to a likelihood for an existing extension $\left(\mathrm{L}_{\text {ext }}\right)$, $\mathrm{TS}_{\text {ext }}=2 \log \left(\mathrm{L}_{e x t} / \mathrm{L}_{p t}\right)$. The 'Extension Width' which is the $68 \%$ containment radius of the extension model $\left(\mathrm{R}_{68}\right)$, was found to be $0^{\circ} .6107+0^{\circ} .0866-0^{\circ} .1141$, with a $\mathrm{TS}_{\text {ext }}$ value of $\sim 40$ for the radial Gaussian model. As an extended source, PS J1934.5+1845 was detected with a significance of $\sim 13 \sigma$ (i.e. $\mathrm{TS}=168$ ).

\subsection{Spectral Measurements}

The spectrum was fit to PL model, where the spectral index is found to be $\Gamma=2.98 \pm 0.09$. The total photon flux and energy flux was found to be $(2.99 \pm 0.31) \times 10^{-9}$ photons cm $^{-2} \mathrm{~s}^{-1}$ and $(1.20 \pm 0.11) \times 10^{-6} \mathrm{MeV} \mathrm{cm}^{-2} \mathrm{~s}^{-1}$, respectively, for the point-like source model having a PL-type spectrum. Assuming a PL-type spectrum for this extended source, we obtained $\Gamma=2.38 \pm$ 0.07 , and the total photon flux and energy flux of PS J1934.5+1845 was found to be (2.54 \pm 0.23$)$ $\times 10^{-8}$ photons $\mathrm{cm}^{-2} \mathrm{~s}^{-1}$ and $(1.74 \pm 0.16) \times 10^{-5} \mathrm{MeV} \mathrm{cm}^{-2} \mathrm{~s}^{-1}$, respectively. The spectrum for the extended emission of PS J1934.5+1845 is shown in Figure 2.

\section{Conclusion \& Outlook}

We detected a new source, PS J1934.5+1845, at about $1^{\circ} .8$ away from 3C 400.2, having a significance of $\sim 13 \sigma$. PS J1934.5+1845 was found to have a radial Gaussian type extension with a radius of $\sim 0^{\circ} .61$. We investigated this source as a part of our analysis, due to the possibility that it might have been contributing to the gamma-ray emission of 3C 400.2. After fitting the extension and checking the TS value of 3C 400.2, we found out that the emission of PS J1934.5+1845 is not directly affecting the gamma-ray emission of 3C 400.2. The gamma-ray emission of PS J1934.5+1845 needs to be further investigated by modeling the SED in order to understand whether the dominating gamma-ray emission mechanism is leptonic or hadronic.

As a next step, we plan for multi-waveband observations (radio, optical, and X-rays). In addition, we will re-analyze the gamma-ray data to do more variability checks and investigate the energy-dependent source morphology.

\section{References}

[1] Acero, F., et al. (Fermi Collaboration), Fermi Large Area Telescope Third Source Catalog, AJSS, 218, $41(2015)$

[2] Acero F. et al. (Fermi Collaboration), 3FHL: The Third Catalog of Hard Fermi-LAT Sources, AJSS, 232, 23 (2017)

[3] Ergin, T., Sezer, A., Sano, H., Yamazaki, R., \& Fukui, Y., Recombining Plasma and Gamma-Ray Emission in the Mixed-morphology Supernova Remnant 3C 400.2, ApJ, 842, 22 (2017)

[4] Mattox, J. R., Bertsch, D. L., Chiang, J., et al., The Likelihood Analysis of EGRET Data, ApJ, 461, 396 (1996)

[5] Rho, J. \& Petre, R., Mixed-Morphology Supernova Remnants, ApJL, 503, L167 (1998) 